\title{
Generalized permutohedra, $h$-vectors of cotransversal matroids and pure O-sequences
}

\author{
Suho Oh \\ Department of Mathematics \\ University of Michigan \\ Michigan, U.S.A. \\ suhooh@math.umich.edu
}

Submitted: Oct 3, 2012; Accepted: July 27, 2013; Published: Aug 9, 2013

Mathematics Subject Classifications: 05A19, 52B40

\begin{abstract}
Stanley has conjectured that the $h$-vector of a matroid complex is a pure Osequence. We will prove this for cotransversal matroids by using generalized permutohedra. We construct a bijection between lattice points inside an $r$-dimensional convex polytope and bases of a rank $r$ transversal matroid.
\end{abstract}

\section{Introduction}

Matroids, simplicial complexes and their $h$-vectors are all interesting objects that are of great interest in algebraic combinatorics and combinatorial commutative algebra. An order ideal is a finite collection $X$ of monomials such that, whenever $M \in X$ and $N$ divides $M$, then $N \in X$. If all maximal monomials of $X$ have the same degree, then $X$ is pure. A pure $O$-sequence is the vector, $h=\left(h_{0}=1, h_{1}, \ldots, h_{t}\right)$, counting the monomials of $X$ in each degree. The following conjecture by Stanley has motivated a great deal of research on $h$-vectors of matroid complexes:

Conjecture 1. The $h$-vector of a matroid is a pure O-sequence.

The above conjecture has been proven for cographic matroids by both Merino [7] and Chari [3]. It also has been proven for lattice-path matroids by Schweig [11]. Lattice path matroids are special cases of cotransversal matroids, and we will prove the conjecture for cotransversal matroids. We would also like to note that there has been plenty of interesting results related to this conjecture: $[1],[2],[5],[6],[9],[13],[14]$. 
We prove the conjecture for cotransversal matroids by associating a polytope to each cotransversal matroid. The lattice points inside this polytope will be in bijection with bases of the matroid, and will naturally induce a pure order ideal we are looking for.

In section 2, we will go over the properties of transversal matroids. In section 3 , the properties of generalized permutohedra will be reviewed. In section 4 , we show a connection between transversal matroids and generalized permutohedra. In section 5, we prove our main result.

\section{Preliminaries on matroids}

In this section, we will provide some notation and tools on transversal matroids that we are going to use throughout the paper.

Definition 2 ([8]). Let $E$ be a set and let $\mathcal{M}$ be a non-empty collection of subsets of $E$ such that the following condition is satisfied: if $B_{1}$ and $B_{2}$ are members of $\mathcal{M}$ and $x \in B_{1} \backslash B_{2}$, then there is an element $y$ of $B_{2} \backslash B_{1}$ such that $\left(B_{1} \backslash\{x\}\right) \cup\{y\} \in \mathcal{M}$. Then $\mathcal{M}$ is called a matroid, and each element of $\mathcal{M}$ is called a base of $\mathcal{M}$.

It is a well known fact that all bases of a matroid have the same cardinality, and that number is called the rank of the matroid. The set of bases forms a collection of facets of a pure simplicial complex, and the $h$-vector of a matroid is defined as the $h$-vector of the complex. In the next paragraph, we review a method of computing the $h$-vector of a matroid by a certain degree counting of the bases, and we will use that as the definition of the $h$-vector in this paper. Throughout the paper, unless stated otherwise, a matroid $\mathcal{M}$ will be a rank $r$ matroid over the ground set $[\bar{n}]:=\{\overline{1}<\cdots<\bar{n}\}$.

An element $i$ of a base $B$ is internally active if $(B \backslash\{i\}) \cup\{j\}$ is not a base for any $j<i$. An element $e \notin B$ is externally active if $(B \cup\{e\}) \backslash\{j\}$ is a not a base for all $j>e$. If an element not in $B$ is not externally active with respect to $B$, we say that it is externally passive with respect to $B$. We denote $e_{\mathcal{M}}(B)$ to count the number of such elements.

Lemma 3 ([11],[16]). Let $\left(h_{0}, \cdots, h_{r}\right)$ be the $h$-vector of a matroid $\mathcal{M}$. For $0 \leqslant i \leqslant r$, $h_{i}$ is the number of bases of $\mathcal{M}$ with $r-i$ internally active elements.

The dual matroid $\mathcal{M}^{*}$ of a matroid $\mathcal{M}$ is a collection of bases which are complements to the bases of $\mathcal{M}$.

Remark 4 . The way we will view $h_{i}$ in this paper is to count the number of bases in the dual-matroid of $\mathcal{M}$ with $i$ externally passive elements.

In this paper, we will be focusing on a particular class of matroids coming from bipartite graphs, called transversal matroids. Let $\mathcal{A}$ be a family $\left(A_{1}, \ldots, A_{r}\right)$ of subsets of the set $L=\{\overline{1}, \ldots, \bar{n}\}$. Then the bipartite graph $\mathcal{G}(\mathcal{A})$ associated with $\mathcal{A}$ has vertex set $L$ and $R=\{1, \ldots, r\}$ with edge set given by $\left\{(a, b) \mid a \in L, b \in R\right.$ and $\left.a \in A_{b}\right\}$. Throughout the paper, we will call the vertex set $L$ and $R$ of a bipartite graph as the set of left vertices and the set of right vertices respectively. 
Given a subgraph $T$ of this graph, let $l t(T)$ denote the subset of vertices of $L$ covered by edges of $T$ and let $r t(T)$ denote the subset of vertices of $R$ covered by edges of $T$. Then collection of $l t(T)$ for all maximal matchings of $\mathcal{G}(\mathcal{A})$ form the set of bases of a matroid. We denote this matroid by $\mathcal{M}(\mathcal{A})$. If $\mathcal{M}$ is an arbitrary matroid and $\mathcal{M} \cong \mathcal{M}(\mathcal{A})$ for some family $\mathcal{A}$ of sets, then $\mathcal{M}$ a transversal matroid and $\mathcal{A}$ is a presentation of $\mathcal{M}$.

In Figure 1, we have a presentation of a family $(\{\overline{1}, \overline{4}, \overline{5}, \overline{6}, \overline{7}, \overline{8}\},\{\overline{2}, \overline{3}, \overline{6}, \overline{7}, \overline{8}\})$.

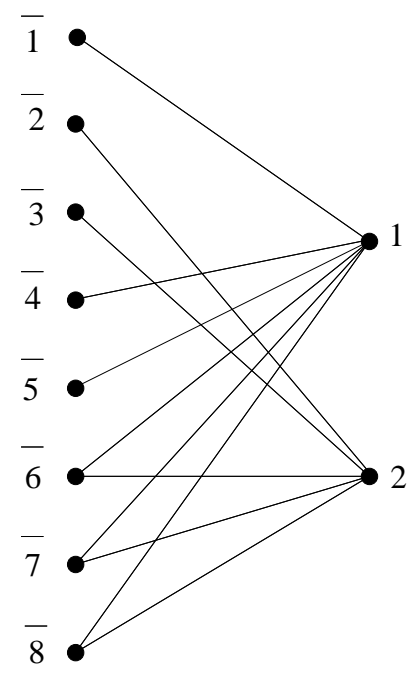

Figure 1 - A bipartite graph

A cotransversal matroid is a matroid that is the dual matroid of some transversal matroid. We now state the main result of this paper:

Theorem 5. The h-vector of a cotransversal matroid is a pure O-sequence. In other words, Stanley's conjecture is true for cotransversal matroids.

In the remaining part of the section, we go over two essential tools we need to work with transversal matroids. The following lemma is given as an exercise in [8].

Lemma 6 ([8]). Let $\mathcal{M}$ be a transversal matroid that has rank $r$. Then there exists a presentation of $\mathcal{M}$ that has exactly $r$ members.

Given a vertex $v$ inside a graph $G$, we will use $N(v)$ to denote the set of neighbors of $v$.

Theorem 7 ([8], [4]). Let $I_{1}, \cdots, I_{r} \subseteq[r]$. The following conditions are equivalent:

1. For any $S \subseteq[r]$ we have $\left|\bigcup_{i \in S} I_{i}\right| \geqslant|S|$.

2. There exists a bijection $f$ from $[r]$ to $[r]$ such that for all $t \in[r], f(t) \in I_{t}$.

The first condition is called the Hall's marriage condition, and the bijection $f$ in the second condition is referred to as the system of distinct representatives.

A subset $H=\left\{h_{1}, \ldots, h_{r}\right\}$ of $L$ is a base of $\mathcal{M}(\mathcal{A})$ if and only if $N\left(h_{1}\right), \ldots, N\left(h_{r}\right)$ satisfies the Hall's Marriage condition. 


\section{$3 \quad$ Generalized permutohedra}

In this section, we review generalized permutohedra and study some properties of spanning trees that we will be using in this paper. The content related to generalized permutohedra follows that of $[10]$.

Definition 8 ([10]). Let $d$ be the dimension of the Minkowski sum $P_{1}+\cdots+P_{n}$, where $P_{1}, \ldots, P_{n}$ are convex polytopes. A Minkowski cell in this sum is a polytope $B_{1}+\cdots+B_{n}$ of dimension $d$ where $B_{i}$ is the convex hull of some subset of vertices of $P_{i}$. A mixed subdivision of the sum is the decomposition into union of Minkowski cells such that intersection of any two cells is their common face. A mixed subdivision is fine if for all cells $B_{1}+\cdots+B_{n}$, all $B_{i}$ are simplices and $\sum \operatorname{dim} B_{i}=d$.

Remark 9. All mixed subdivisions in our paper, unless otherwise stated, will be referring to fine mixed subdivisions.

Let $G \subseteq K_{n, r+1}$ be a bipartite graph with no isolated vertices. Label the set of left vertices using $\overline{1}, \ldots, \bar{n}$, and label the set of right vertices using $0, \ldots, r$. We will use $[\hat{r}]$ to denote the set $\{0,1, \cdots, r\}$. Let us associate $G$ with the collection $\mathcal{I}_{G}$ of subsets $I_{1}, \cdots, I_{n} \subseteq[\hat{r}]:=\{0,1, \cdots, r\}$ such that $j \in I_{i}$ if and only if there is an edge in $G$ which connects a left vertex labeled $\bar{i}$ with a right vertex labeled $j$.

Definition 10. Let $e_{0}, \ldots, e_{r}$ be the coordinate vectors of $\mathbb{R}^{r+1}$. The generalized permutohedron $P_{G}$ is defined as the Minkowski sum

$$
P_{G}=\Delta_{I_{1}}+\cdots+\Delta_{I_{n}}
$$

where $\Delta_{I}$ is defined to be to be the convex hull of points $e_{i}$ for $i \in I$.

Remark 11 ([10]). This polytope is a special case of the family of polytopes that can be defined by:

$$
\left\{\left(t_{0}, \ldots, t_{r}\right) \in \mathbb{R}^{r+1} \mid \sum_{i=0}^{r} t_{i}=z_{[\hat{r}]}, \sum_{i \in I} t_{i} \geqslant z_{I}\right\}
$$

Proposition $12([10])$. Let $I_{1}, \cdots, I_{r} \subseteq[\hat{r}]$. The following conditions are equivalent:

1. For any distinct $i_{1}, \cdots, i_{k}$, we have $\left|I_{i_{1}} \cup \cdots \cup I_{i_{k}}\right| \geqslant k+1$.

2. For any $j \in[\hat{r}]$, there is a system of distinct representatives in $I_{1}, \cdots, I_{r}$ that avoids $j$.

The above condition is called the dragon marriage condition.

There is a nice connection between Hall's marriage condition and the dragon marriage condition.

Remark 13 . When $H_{1}, \ldots, H_{n}$ are subsets of $[r]$, they satisfy Hall's marriage condition if and only if $\{0\} \cup H_{1}, \ldots,\{0\} \cup H_{n}$ satisfy the dragon marriage condition. 
Definition 14 ([10]). Let us say that a sequence of nonnegative integers $\left(a_{1}, \cdots, a_{n}\right)$ is a $G$-draconian sequence if $\sum a_{i}=r$ and for any subset $\left\{i_{1}<\cdots<i_{k}\right\} \subseteq[n]$, we have $\left|I_{i_{1}} \cup \cdots \cup I_{i_{k}}\right| \geqslant a_{i_{1}}+\cdots+a_{i_{k}}+1$. Equivalently, if the sequence $I_{1}^{a_{1}}, \cdots, I_{n}^{a_{n}}$, where $I^{a}$ means $I$ repeated $a$ times, satisfies the dragon marriage condition.

An important property of generalized permutohedra is that fine Minkowski cells can be described by spanning trees of $G$. For a sequence of nonempty subsets $\mathcal{J}=\left(J_{1}, \cdots, J_{n}\right)$, let $G_{\mathcal{J}}$ be the graph with edges $(\bar{i}, j)$ for $j \in J_{i}$.

Lemma 15 ([10]). Each fine mixed cell in a mixed subdivision of $P_{G}$ has the form $\Delta_{J_{1}}+$ $\cdots \Delta_{J_{n}}$, for some sequence of nonempty subsets $\mathcal{J}=\left(J_{1}, \cdots, J_{n}\right)$ in $[\hat{r}]$ such that $G_{\mathcal{J}}$ is a spanning tree of $G$.

Remark 16. As noted in [10], the above lemma implies that each fine mixed cell $\Delta_{J_{1}}+$ $\cdots \Delta_{J_{n}}$ is isomorphic to the direct product of simplices $\Delta_{J_{1}} \times \cdots \times \Delta_{J_{n}}$. By choosing any $j$ inside $J_{i}$ with $\left|J_{i}\right|>1$, the product $\Delta_{J_{1}} \times \cdots \Delta_{J_{i} \backslash\{j\}} \times \cdots \Delta_{J_{n}}$ describes a facet of the cell $\Delta_{J_{1}} \times \cdots \times \Delta_{J_{n}}$. Moreover, any facet is of such format.

Given a spanning tree $T$ of $G$, we denote $\prod_{T}$ to be the corresponding Minkowski cell $\Delta_{J_{1}}+\cdots+\Delta_{J_{n}}$. We can say a bit more about the lattice points in each $\prod_{T}$ :

Proposition 17 ([10]). Any integer lattice point of a fine Minkowski cell $\prod_{G_{\mathcal{J}}}$ in $P_{G}$ is of form $p_{1}+\cdots+p_{n}$ where $p_{i}$ is an integer lattice point in $\Delta_{J_{i}}$.

Given any subgraph $T$ of $G$, define the left degree vector $l d(T)=\left(d_{\overline{1}}, \cdots, d_{\bar{n}}\right)$ where $d_{\bar{i}}$ is the degree of the vertex $\bar{i}$ in $T$ minus 1 . Similarly, we define the right degree vector $r d(T)=\left(d_{0}, \cdots, d_{r}\right)$ where $d_{j}$ is the degree of the vertex $j$ in $T$ minus 1 . The following proposition is stated in the proof of Theorem 11.3 in [10].

Proposition 18 ([10]). Consider a fine mixed subdivision $\left\{\prod_{T_{1}}, \cdots, \prod_{T_{s}}\right\}$ of the polytope $P_{G}$. Then the map $\prod_{T_{i}} \rightarrow l d\left(T_{i}\right)$ is a bijection between fine cells $\prod_{T_{i}}$ in this subdivision and $G$-draconian sequences.

For two spanning trees $T$ and $T^{\prime}$ of $G$, let $U\left(T, T^{\prime}\right)$ be the directed graph which is the union of edges of $T$ and $T^{\prime}$ with edges of $T$ oriented from left to right and edges of $T^{\prime}$ oriented from right to left. A directed cycle is a sequence of directed edges $\left(i_{1}, i_{2}\right),\left(i_{2}, i_{3}\right), \cdots,\left(i_{k-1}, i_{k}\right),\left(i_{k}, i_{1}\right)$ such that all $i_{1}, \cdots, i_{k}$ are distinct.

Lemma 19 ([10]). For two spanning trees $T, T^{\prime}$, the corresponding Minkowski cells can be in the same mixed subdivision only if $U\left(T, T^{\prime}\right)$ has no directed cycles of length $\geqslant 4$.

We will say that $T, T^{\prime}$ are compatible if it satisfies the condition of Lemma 19, and incompatible if not.

Before we end, we will state some basic facts about spanning trees of bipartite graphs that we will be using. Recall that we are assuming $G$ to be a bipartite graph inside $K_{n, r+1}$. Let us add in the extra assumption that $n \geqslant r$. Let $T$ be a spanning tree of $G$. We use $L D_{T}(I)$ to denote the sum of $d_{i}$ 's for $i \in I$, where $l d(T)=\left(d_{1}, \cdots, d_{n}\right)$. We use $N_{T}(I)$ to denote the set of neighbors of vertex set $I$ inside $T$. 
Lemma 20. Let $T$ be any spanning tree of $G \subseteq K_{n, r+1}$, where $n \geqslant r$. Set $I$ to be a subset of left vertices such that $|I|<n$. Then $\left|N_{T}(I)\right| \geqslant L D_{T}(I)+1$. Let $J$ be a subset of right vertices such that $|J|<r+1$. Then $L D_{T}\left(N_{T}(J)\right) \geqslant|J|$.

Proof. The first claim follows directly from the fact that $T$ is a spanning tree. For the second claim, consider the induced subgraph $S$ of $T$ by looking at the vertices $J \cup N_{T}(J) \cup$ $N_{T}\left(N_{T}(J)\right)=N_{T}(J) \cup N_{T}\left(N_{T}(J)\right)$. Let $c$ be the number of connected components of $S$, and divide $J$ into $J_{1}, \ldots, J_{c}$ such that for each $i$, the union $N_{T}\left(J_{i}\right) \cup N_{T}\left(N_{T}\left(J_{i}\right)\right)$ is the set of vertices of a connected component $S_{i}$. For each $i$, we have $\left|N_{T}\left(N_{T}\left(J_{i}\right)\right)\right|>\left|J_{i}\right|$ since $T$ is a spanning tree.

In general, given a spanning tree $T$ of $K_{n, r}$, we have $L D_{T}([n])=r-1$ since the total number of edges is $n+r-1$. The component $S_{i}$ is a spanning tree with left vertex set $N_{T}\left(J_{i}\right)$ and right vertex set $N_{T}\left(N_{T}\left(J_{i}\right)\right)$. Hence we get $L D_{T}\left(N_{T}\left(J_{i}\right)\right)=$ $L D_{S_{i}}\left(N_{T}\left(J_{i}\right)\right)=\left|N_{T}\left(N_{T}\left(J_{i}\right)\right)\right|-1 \geqslant\left|J_{i}\right|$. Summing the inequalities for all components $S_{i}$, we get $L D_{T}\left(N_{T}(J)\right) \geqslant|J|$.

Lemma 21. Let $T$ and $T^{\prime}$ be spanning trees of $G \subseteq K_{n, r+1}$, where $n \geqslant r$. Denote the left-degree vector of $T$ as $\left(d_{1}, \cdots, d_{n}\right)$ and the left-degree vector of $T^{\prime}$ as $\left(d_{1}^{\prime}, \cdots, d_{n}^{\prime}\right)$. If after some relabeling of the set $[n]$,

- $d_{n}<d_{n}^{\prime}$ and $d_{1}>d_{1}^{\prime}$,

- $d_{i} \geqslant d_{i}^{\prime}$ for all $i \neq n$,

- 0 is connected to $\overline{1}, \bar{n}$ via an edge in $T^{\prime}$,

- 0 is connected to $\bar{n}$ via an edge in $T$,

then $T$ and $T^{\prime}$ are incompatible.

Proof. Let $H$ be a subset of right vertices of $G$ such that $\bar{n} \notin N_{T^{\prime}}(H)$. Using Lemma 20, we get $\left|N_{T}\left(N_{T^{\prime}}(H)\right)\right| \geqslant L D_{T}\left(N_{T^{\prime}}(H)\right)+1$. From the way that $T$ and $T^{\prime}$ was constructed, we get $L D_{T}\left(N_{T^{\prime}}(H)\right) \geqslant L D_{T^{\prime}}\left(N_{T^{\prime}}(H)\right)$. By applying Lemma 20 again, we get $L D_{T^{\prime}}\left(N_{T^{\prime}}(H)\right) \geqslant|H|$, from which we can conclude that $\left|N_{T}\left(N_{T^{\prime}}(H)\right)\right|>|H|$. Notice that if $N_{T^{\prime}}(H)$ contains $\overline{1}$, then we have $L D_{T}\left(N_{T^{\prime}}(H)\right)>L D_{T^{\prime}}\left(N_{T^{\prime}}(H)\right)$, and hence we get $\left|N_{T}\left(N_{T^{\prime}}(H)\right)\right|>|H|+1$.

Assume that $T$ and $T^{\prime}$ are compatible for the sake of contradiction. We have an edge $(\bar{n}, 0)$ in $T$ and $(\overline{1}, 0)$ in $T^{\prime}$. Denote $H_{1}$ to be $N_{T}(\overline{1}) \backslash\{0\}$. To prevent an alternating cycle of length greater than 4 in $U\left(T, T^{\prime}\right)$, we have $\bar{n} \notin N_{T^{\prime}}\left(H_{1}\right)$. According to the argument in the previous paragraph, $H_{2}:=N_{T}\left(N_{T^{\prime}}\left(H_{1}\right)\right) \backslash\{0\}$ is strictly larger than $H_{1}$. Again, to prevent an alternating cycle of length greater than 4 in $U\left(T, T^{\prime}\right)$, we have $\bar{n} \notin N_{T^{\prime}}\left(H_{2}\right)$. By repeating this procedure, setting $H_{i+1}$ to be $N_{T}\left(N_{T^{\prime}}\left(H_{i}\right)\right) \backslash\{0\}$ in each step, this goes on and on, contradicting the fact that number of vertices in $G$ is finite. 


\section{Lattice points of $P_{\mathcal{M}}$ and bases of $\mathcal{M}$.}

In this section, given a transversal matroid $\mathcal{M}$, we construct a generalized permutohedron $P_{\mathcal{M}}$ from it. Moreover, we show that any fine mixed subdivision of $P_{\mathcal{M}}$ induces a bijection between the bases of $\mathcal{M}$ and lattice points of $P_{\mathcal{M}}$ lying inside the region satisfying $x_{i} \geqslant 1$ for all $i \in[r]$.

Let $\mathcal{M}$ be a transversal matroid of rank $r$ over the base set $[\bar{n}]=\{\overline{1}, \ldots, \bar{n}\}$. Then by Lemma 6 , there is a bipartite graph that gives a presentation of $\mathcal{M}$, and is a subgraph of the complete bipartite graph $K_{n, r}$. As before, we label the set of left vertices by $\overline{1}, \ldots, \bar{n}$ and label the set of right vertices by $1, \ldots, r$. Now we add a vertex labeled 0 and connect it to all left vertices to get a new bipartite graph $G$. Then we define $P_{\mathcal{M}}$ to be the generalized permutohedron $P_{G}$.

As before, we use $I_{1}, \ldots, I_{n}$ to denote $N(\overline{1}), \ldots, N(\bar{n})$. One property to keep an eye on is that 0 is contained in all of those sets. Our strategy for showing Stanley's conjecture is to assign a bijection between bases of $\mathcal{M}$ and lattice points of $P_{\mathcal{M}}$ that satisfy $x_{1}, \ldots, x_{r} \geqslant 1$.

Given a generalized permutohedron $P$, let $p$ be an integer lattice point of $P$. We say that $p$ is a base point of $P$ if $p+\epsilon \mu$ is a point inside $P$ for a very small positive number $\epsilon$, where $\mu$ is defined to be $r e_{0}-\sum_{i=1}^{r} e_{i}$.

Lemma 22. Base points of $P_{\mathcal{M}}$ are exactly the integer lattice points of $P_{\mathcal{M}}$ that satisfy $x_{1}, \ldots, x_{r} \geqslant 1$.

Proof. All summands of $P_{\mathcal{M}}$ are simplices which contain the vertex $e_{0}$. Let $p_{0}$ be the unique vertex of $P_{\mathcal{M}}$ given by the coordinate $(n, 0, \ldots, 0)$. By Remark 16, each facet surrounding $p_{0}$ is on a hyperplane $x_{i}=0$ for some $i \in[r]$. The integer lattice points of $P_{\mathcal{M}}$ that are not base points, are exactly the points on those facets.

Let $\prod_{\mathcal{J}}=\Delta_{J_{1}} \times \cdots \times \Delta_{J_{n}}$ be some Minkowski cell inside a mixed subdivision of $P_{\mathcal{M}}$.

Definition 23. If for all $i \in[n]$, we have $\left|J_{i}\right| \leqslant 2$, we say that $\prod_{\mathcal{J}}$ is zonotopal.

Lemma 24. A Minkowski cell $\prod_{\mathcal{J}}$ inside a mixed subdivision of $P_{\mathcal{M}}$ contains a base point of $\prod_{\mathcal{J}}$ if and only if $\prod_{\mathcal{J}}$ is zonotopal.

Proof. We first show that if $\prod_{\mathcal{J}}$ is zonotopal, it contains a base point of $\prod_{\mathcal{J}}$. We construct a subgraph $T$ of $K_{r+1}$ by collecting the edges $(a, b)$ for each $J_{i}=\{a, b\}$. Then $T$ is a spanning tree since $G_{\mathcal{J}}$ is a spanning tree of $K_{n, r+1}$. Think of $T$ as a rooted tree having 0 as the root. For each $J_{i}=\{a, b\}$, where $b$ is a descendant of $a$, set $q_{i}$ to be $e_{a}$ and $p_{i}$ to be $e_{b}$. Let $l_{i}$ denote the number of descendants of $a$ inside $T$. Consider the point $p=\sum p_{i}$. We will show that this is a base point of $\prod_{\mathcal{J}}$. For each $i \in[n]$, the point $p_{i}+l_{i} \epsilon\left(q_{i}-p_{i}\right)$ is inside $\Delta_{J_{i}}$. Therefore, we can conclude that $p+\epsilon \sum l_{i}\left(q_{i}-p_{i}\right)=p+\epsilon \mu$ is a point inside $\prod_{\mathcal{J}}$

We now show that for $\prod_{\mathcal{J}}$ to contain a base point, $\prod_{\mathcal{J}}$ has to be zonotopal. Let $p=p_{1}+\cdots+p_{n}$ be the base point of $\prod_{\mathcal{J}}$, where $p_{i} \in \Delta_{J_{i}}$. The point $p$ being a base point implies that we can decrease the value of $a$-th coordinate from $p$ and still stay in $\prod_{\mathcal{J}}$ for all $a \in[r]$. In order for this to be true, for each $a \in[r]$, there has to exist $b \in[n]$ such 
that we can decrease the value of $a$-th coordinate from $p_{b}$ and still stay in $\Delta_{J_{b}}$. But given any $p_{b} \in \Delta_{J_{b}}$, there is exactly one coordinate $a \in[r]$ where we can decrease its value and still stay in $\Delta_{J_{b}}$ if $\left|J_{b}\right| \geqslant 2$, and none otherwise. Therefore, we need at least $r J_{i}$ 's having cardinality $\geqslant 2$, and this implies that $\prod_{\mathcal{J}}$ is zonotopal.

Remark 25. From the above proof, it is easy to see that the coordinate of the base point is only affected by $J_{i}$ 's such that $\left|J_{i}\right|=1$. More precisely, the coordinate of the point is given by $\left(n-r-x_{1}-\cdots-x_{r}, x_{1}+1, \ldots, x_{r}+1\right)$, where $x_{k}$ counts the number of times $k$ appears among $J_{i}$ 's having cardinality 1 .

Proposition 26. There is a bijection between base points of $P_{\mathcal{M}}$ and bases of $\mathcal{M}$.

Proof. Given a fixed fine mixed subdivision of $P_{\mathcal{M}}$, Proposition 18 and Remark 13 tells us that there is a bijection between zonotopal cells of $P_{\mathcal{M}}$ and bases of $\mathcal{M}$. All we need to show is that every base point of $P_{\mathcal{M}}$ is a base point of some zonotopal cell.

The facets of possible cells of $P_{\mathcal{M}}$ are of form $\sum_{i \in I} x_{i}=z_{I}$ for some subset $I$ of $\{0\} \cup[r]$. This means that none of the facets are parallel to the vector $\mu$, which implies that $p+\epsilon \mu$ is in the interior of some cell which contains $p$ on its hull. This cell has to be zonotopal by Lemma 24 .

We have seen that each fine mixed subdivision of $P_{\mathcal{M}}$ induces a bijection between base points of $P_{\mathcal{M}}$ and bases of $\mathcal{M}$. In the next section, we come up with a fine mixed subdivision such that $n-r-x_{0}$ of a base point equals the externally passive degree of the corresponding base in $\mathcal{M}$.

\section{Lexicographical subdivision of $P_{\mathcal{M}}$.}

In this section, we want to find a fine mixed subdivision of $P_{\mathcal{M}}$ such that if we use the bijective map defined in the previous section to associate the bases to the lattice points of $P_{\mathcal{M}}$, the externally passive degree can be read off by looking at the sum of all coordinates except 0 .

We use the fact that the fine mixed subdivision of a generalized permutohedron is related to a triangulation of certain polytope via the Cayley trick. Let $e_{\overline{1}}, \ldots, e_{\bar{n}}$, $e_{0}, e_{1}, \ldots, e_{r}$ be the standard basis of $\mathbb{R}^{n+r+1}$. Embed the space $\mathbb{R}^{r+1}$ where the polytopes $\Delta_{I}$ live for $I \subseteq[\hat{r}]$. As before, let $I_{i}$ denote the collection of $j$ 's such that $(\bar{i}, j)$ is an edge of $G$. The root polytope $Q_{G}$ is defined as the convex hull of the vertices $e_{i}+e_{j}$ 's, for each edge $(\bar{i}, j)$ of $G$.

Lemma 27 ([10]). Fine mixed subdivisions of $P_{G}$ are in one-to-one correspondence with triangulations of $Q_{G}$. A fine mixed cell in $P_{G}$ given by $\Delta_{J_{1}} \times \cdots \times \Delta_{J_{n}}$ corresponds to a simplex which has vertices $e_{\bar{i}}+e_{j}$ for each pair $(\bar{i}, j)$ satisfying $j \in J_{i}$.

Let $P_{G}$ be a generalized permutohedron and $P_{G^{\prime}}$ be $P_{G}+\Delta_{J}$, where $0 \in J$. In other words, $G^{\prime}$ is a bipartite graph obtained by adding a left vertex $v$ with neighborhood $J$ 
to the bipartite graph $G$. Start from a triangulation of $Q_{G}$. This naturally induces a triangulation on the cone formed by $e_{v}+e_{0}$ and $Q_{G}$. This cone is a convex subpolytope of $Q_{G^{\prime}}$, so we can extend the triangulation of the cone to a triangulation of $Q_{G^{\prime}}$. We say that such triangulation of $Q_{G^{\prime}}$ is obtained by extending the triangulation of $Q_{G}$ in direction 0 . For the corresponding mixed subdivisions, we say that the mixed subdivision of $P_{G^{\prime}}$ is obtained by extending the mixed subdivision of $P_{G}$ in direction 0 . One can see that for each cell $\prod_{T}$ in the mixed subdivision of $P_{G}, \prod_{T}+\Delta_{\{0\}}$ is a cell inside the extended mixed subdivision of $P_{G^{\prime}}$. We will use this extension method to define a lexicographical subdivision of $P_{G}$.

Start from a Minkowski sum $X_{0}:=\Delta_{\{0,1\}}+\cdots+\Delta_{\{0, r\}}$. We use $X_{i}$ to denote the sum $X_{0}+P_{i}$, where $P_{i}$ is the sum $\Delta_{I_{1}}+\cdots+\Delta_{I_{i}}$. We start from a subdivision of $X_{0}$, which is unique, and repeat the process of extending the subdivision in direction 0 to obtain a subdivision of $X_{i}$ for each $i \in[n]$. We call this a lexicographical subdivision of $X_{i}$.

Lemma 28. Let $\prod_{T}$ be a cell inside a lexicographical subdivision of $X_{i}$, for $i \geqslant 1$. Then $0 \in T_{r+i}$.

Proof. We will use induction on the size of $\left|T_{r+i}\right|$. When $\left|T_{r+i}\right|=1$, the cell $\prod_{T}$ has leftdegree vector $\left(\left|T_{1}\right|-1, \ldots,\left|T_{r+i-1}\right|-1,0\right)$. Proposition 18 implies that there is some cell $\prod_{T^{\prime}}$ that has left degree vector $\left(\left|T_{1}\right|-1, \ldots,\left|T_{r+i-1}\right|-1\right)$ in $X_{i-1}$. From the definition of lexicographical subdivision, there is a cell corresponding to the tree $\left(T_{1}^{\prime}, \ldots, T_{r+i-1}^{\prime},\{0\}\right)$ in $X_{i}$. Since this cell has the same left degree vector as $\prod_{T}$, Proposition 18 tells us that $T_{r+i}=\{0\}$.

Now assume for the sake of induction that $0 \in J_{r+i}$ for all cells $\prod_{\mathcal{J}}$ such that $\left|J_{r+i}\right|<$ $\left|T_{r+i}\right|$. There is some $q \in T_{r+i}$ such that by crossing the facet $\Delta_{T_{1}}+\cdots+\Delta_{T_{r+i} \backslash\{q\}}$, we reach another cell $\prod_{S}$ in $X_{i}$. From Proposition 18, we have $\left|S_{r+i}\right|<\left|T_{r+i}\right|$. By the induction hypothesis, we have $0 \in S_{r+i}$. Since $T_{r+i} \backslash\{q\}=S_{r+i}$, we get $0 \in T_{r+i}$.

Let $\prod_{T}$ be a cell inside a lexicographical subdivision of $X_{i}$, that intersects the region $0<x_{j}<1$ and does not lie inside $X_{i-1}+\Delta_{\{0\}}$. Writing $T=\left(T_{1}, \ldots, T_{r+i}\right)$, we can see that $T_{j} \neq\{j\}$, since otherwise the cell will not intersect with the region $0<x_{j}<1$. By comparing this cell to the cell $\prod_{T^{\prime}}$ which is given by $\Delta_{\{0,1\}}+\cdots+\Delta_{\{0, r\}}+\Delta_{\{0\}}+\cdots+\Delta_{\{0\}}$, we can see that $j \notin T_{k}$ for $k>r$, since otherwise we have a length 4 alternating cycle in $U\left(T, T^{\prime}\right)$. But since $T$ is a spanning tree, some $T_{i}$ has to include $j$, which implies that $j \in T_{j}$. Therefore, we can conclude that $T_{j}=\{0, j\}$ and that the cell $\prod_{T}$ is included in the region $0 \leqslant x_{j} \leqslant 1$.

Remark 29. Inside a lexicographical subdivision of $X_{i}$, no cell crosses $x_{j}=1$ for each $j \in[r]$. Each cell $\prod_{T}$ of $X_{i}$ inside the region $x_{j} \geqslant 1$ for all $j \in[r]$ has $T_{j}=\{j\}$ for all $j \in[r]$.

Since no cell crosses $x_{j}=1$ inside a lexicographical subdivision of $X_{i}$, we can cut the subdivision of $X_{i}$ via $x_{j} \geqslant 1$ for all $j \in[r]$ to get a mixed subdivision of $P_{i}$. When $i=n$, we call this a lexicographical subdivision of $P_{G}$.

Now we wish to show that given a lexicographical subdivision of $P_{\mathcal{M}}$, and using the bijection given via Proposition 26, the value $x_{1}+\cdots+x_{r}-r$ of a base point equals 
the externally passive degree of the corresponding base in $\mathcal{M}$. We will use $H_{1}, \ldots, H_{n}$ to denote $N_{G}(\overline{1}) \backslash\{0\}, \ldots, N_{G}(\bar{n}) \backslash\{0\}$. Given a base $B=\left\{b_{1}<\cdots<b_{r}\right\}$ of $\mathcal{M}$, we call the collection of sets $H_{b_{1}}, \ldots, H_{b_{r}}$ to be the type sequence of $B$. Given a collection $\mathcal{H}^{a}=\left\{H_{1}^{a_{1}}, \ldots, H_{n}^{a_{n}}\right\}$ that satisfies Hall's condition, we denote $E P_{\mathcal{M}}\left(\mathcal{H}^{a}\right)$ to denote the collection of $H_{i}$ 's such that there exists $j<i$ for which $\mathcal{H}^{a} \backslash\left\{H_{i}\right\} \cup\left\{H_{j}\right\}$ satisfies Hall's condition. Beware that the collection is considered as a multiset: for example, $\left\{H_{1}^{2}, H_{2}^{0}, H_{3}^{1}\right\} \cup\left\{H_{1}\right\}=\left\{H_{1}^{3}, H_{2}^{0}, H_{3}^{1}\right\}$.

Remark 30. Let the type sequence of $B \in \mathcal{M}$ be $\mathcal{H}^{a}$. Then the point corresponding to $B$ via the bijection in Proposition 26 is the base point of a cell having left degree vector given by $a$.

Now we show that given a cell $\prod_{\mathcal{J}}$ with left degree vector $a$ inside a lexicographical subdivision of $P_{\mathcal{M}}$, there is a connection between whether 0 is in $J_{i}$ or not and whether $H_{i}$ is a member of $E P_{\mathcal{M}}\left(\mathcal{H}^{a}\right)$ or not.

Lemma 31. Let $\mathcal{M}$ be a transversal matroid and $P_{\mathcal{M}}$ be its corresponding generalized permutohedron. Consider a Minkowski cell $\prod_{\mathcal{J}}$, where we write $\mathcal{J}=\left\{J_{1}, \ldots, J_{n}\right\}$, with left degree vector a inside a lexicographical subdivision of $P_{\mathcal{M}}$. We have $0 \notin J_{i}$ if and only if $H_{i} \in E P_{\mathcal{M}}\left(\mathcal{H}^{a}\right)$.

Proof. We first show it is enough to show the claim for $X_{n}$, which was used to define lexicographical subdivision. Let $G^{\prime}$ be the bipartite graph corresponding to $X_{n}$. It has left vertices $\overline{1}, \ldots, \overline{r+n}$, where each vertex $\bar{i}$ for $i \leqslant r$ is connected to right vertices 0 and $i$, and each vertex $\overline{r+i}$ for $i \leqslant n$ is connected to $I_{i}$. We set $\mathcal{M}^{\prime}$ to be the transversal matroid which is represented by $G^{\prime}$, and set $\mathcal{K}=\left(\{1\}, \ldots,\{r\}, H_{1}, \ldots, H_{n}\right)$. It is easy to see that $H_{i} \in E P_{\mathcal{M}}\left(\mathcal{H}^{a}\right)$ if and only if $H_{i}=K_{i+r} \in E P_{\mathcal{M}}\left(\mathcal{K}^{a^{\prime}}\right)$ where $a^{\prime}=\left(0, \ldots, 0, a_{1}, \ldots, a_{n}\right)$. Combining this with Remark 29, we can conclude that to prove the lemma, it is enough to show for $\mathcal{M}^{\prime}$ and $X_{n}$ instead.

We start with $X_{0}$. Since the only cell is $X_{0}$ itself, the claim holds. For the sake of induction, assume that the claim holds for $X_{0}, \ldots, X_{q-1}$. This means that the claim holds for cells of $X_{q}$ with $a_{q}=0$. Again, assume for the sake of induction that the claim holds for cells of $X_{q}$ with left degree vector given by $d$, where $d_{q}<a_{q}$.

Set $a^{\prime}$ to be obtained from $a$ by negating 1 from $a_{q}$. Given the collection $\mathcal{H}^{a^{\prime}}$, use $Q_{a}$ to denote the largest subset $Q$ of $[q-1]$ such that there exists exactly $|Q|$ subsets of $Q$ inside the collection. Such $Q_{a}$ is well defined due to the following reasoning: if $A$ and $B$ are such sets that do not contain each other, there are at least $|A \backslash A \cap B|$ number of subsets of $A$ not contained in $B$. There are at least $|A \cup B|$ number of subsets of $A \cup B$, but this number cannot exceed $|A \cup B|$ since the collection $I^{a}$ satisfies Hall's marriage condition. Hence if $A$ and $B$ are two sets that satisfy the condition, then $A \cup B$ also satisfies the condition.

For $i<q$, if $H_{i} \nsubseteq Q_{a}$, the collection $\mathcal{H}^{a} \backslash\left\{H_{q}\right\} \cup\left\{H_{i}\right\}$ satisfies Hall's marriage condition, and hence $H_{i} \in E P_{\mathcal{M}}\left(\mathcal{H}^{a}\right)$. To see this, for the sake of contradiction, assume there is some distinct $i, i_{1}, \ldots, i_{s}$ such that $\left|H_{i} \cup H_{i_{1}} \cup \cdots \cup H_{i_{s}}\right|=s$. This implies $\left|H_{i_{1}} \cup \cdots \cup H_{i_{s}}\right|=s$ and $H_{i} \subseteq Q_{a}$, which gives us a contradiction.

Therefore, we get some sequence $b$ such that: 
- $b_{q}=a_{q}-1$,

- $b_{i}=a_{i}+1$,

- $b_{j}=a_{j}$ for $j \neq i, q$,

- $\mathcal{H}^{b}$ satisfies the Hall marriage condition.

There exists a cell with left degree vector $b$ due to Remark 13 and Proposition 18. By induction hypothesis, the claim holds for this cell. Using Lemma 21 , we get $0 \notin J_{i}$ since we have $0 \in J_{q}, C_{q}$ due to Lemma 28 . Hence we only need to consider $H_{i}$ 's contained in $Q_{a}$.

By Remark 16, we can cross one of the facets of $\prod_{\mathcal{J}}$ given by $\Delta_{J_{1}}+\cdots+\Delta_{J_{q} \backslash\{i\}}$ to get to another cell inside $X_{q}$. By crossing this facet, we reach a cell $\prod_{\mathcal{C}}$ with left degree vector $c$ such that $c_{q}=a_{q}-1$. The claim holds for $\prod_{\mathcal{C}}$ due to induction hypothesis, and for $\mathcal{H}^{c}$ to also satisfy Hall's marriage condition, we need to have $\left\{H \mid H \in \mathcal{H}^{a}, H \subseteq Q_{a}\right\}=$ $\left\{H \mid H \in \mathcal{H}^{c}, H \subseteq Q_{a}\right\}$. This means that:

- $a_{i}=c_{i}$ for all $i$ such that $H_{i} \subseteq Q_{a}$,

- we have $C_{i}=J_{i}$ for all $i$ such that $H_{i} \subseteq Q_{a}$.

- for $H_{i} \subseteq Q_{a}$, we have $H_{i} \in E P_{\mathcal{M}}\left(\mathcal{H}^{a}\right)$ if and only if $H_{i} \in E P_{\mathcal{M}}\left(\mathcal{H}^{c}\right)$.

Since the lemma holds for $\prod_{\mathcal{C}}$, we have also proven the claim for $\prod_{\mathcal{J}}$. By induction, the claim holds for all cells inside a lexicographical subdivision of $X_{q}$. Again by induction, we have shown that the statement is true for $X_{n}$, and from the argument in the first paragraph, the statement holds for $P_{\mathcal{M}}$.

Let $B$ be a base in $\mathcal{M}$ and $p=\left(c_{0}, \ldots, c_{r}\right) \in P_{\mathcal{M}}$ be the corresponding base point via Proposition 26. Combining Remark 25 and Lemma 31, we can see that $c_{1}+\cdots+c_{r}-r=$ $e_{\mathcal{M}}(B)$.

For each base point at $\left(c_{0}, c_{1}, \cdots, c_{r}\right)$, let us construct a monomial $x_{1}{ }^{c_{1}-1} \cdots x_{r}{ }^{c_{r}-1}$. Then we get a pure monomial order ideal of which Stanley's conjecture is asking for.

Proposition 32. Let $\mathcal{M}$ be a cotransversal matroid. For each base point $\left(c_{1}, \cdots, c_{r}\right)$ in $P_{\mathcal{M}^{*}}$, take a monomial $x_{1}{ }^{c_{1}-1} \cdots x_{r}{ }^{c_{r}-1}$ to form a collection $X$. Then $X$ is a pure monomial order ideal and its degree sequence equals the $h$-vector of $\mathcal{M}$.

Proof. We first show that $X$ is a monomial order ideal. Let $\left(c_{0}, \ldots, c_{r}\right)$ be a point in $P=P_{\mathcal{M}^{*}}$. Let $G$ be the corresponding bipartite graph of $P$. Consider a subgraph we obtain by deleting the right vertex $i$. This gives us a polytope, with one less dimension, and contains $\left(c_{0}, \ldots, 0, \ldots, c_{r}\right)$ as a point, which is obtained from $\left(c_{0}, \ldots, c_{r}\right)$ by setting $c_{i}$ to 0 . The point is also inside $P$, which implies that $\left(c_{0}, \ldots, c_{i}-1, \ldots, c_{r}\right)$ is also inside $P$. This proves that $X$ is a monomial order ideal. 
Now let us show that $X$ is pure. Recall that by Proposition 17, each lattice point of $P$ takes the form $p_{1}+\cdots+p_{n}$, where $p_{i}$ is a lattice point of $\Delta_{I_{i}}$. The point $p_{1}+\cdots+p_{n}$ corresponds to a maximal monomial if and only if $p_{i} \neq e_{0}$ for all $i \in[n]$. This implies that all such points are on the hyperplane $x_{1}+\cdots+x_{r}=n$, from which we can conclude that the corresponding monomials have the same degree.

This implies Stanley's conjecture for cotransversal matroids.

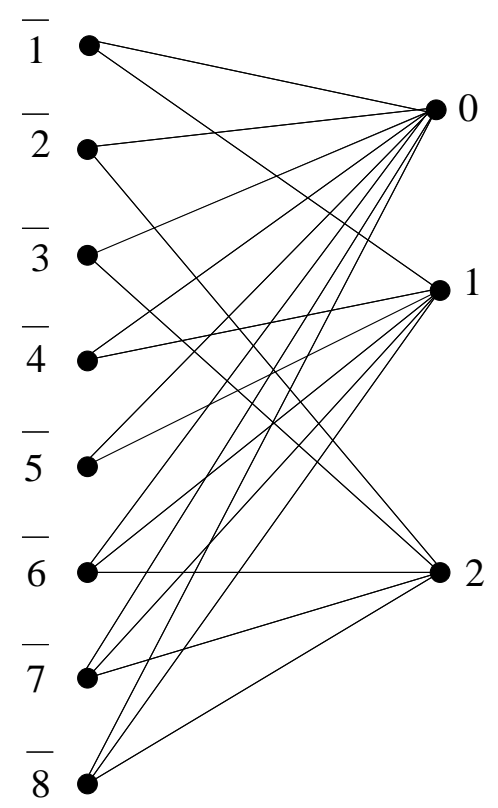

Figure 2 - Padding of the graph given in Figure 1.

Example 33. Let $\mathcal{M}$ be a transversal matroid given by the bipartite graph in Figure 1 . The padded bipartite graph is given Figure 2, and we construct a generalized permutohedron from it. For convenience, we will project down to $x_{0}=0$ to draw the polytope in the $x_{1}, x_{2}$-plane.

First let us consider the cell that lies on the southwest corner. The corresponding summand is given by $\Delta_{\{0,1\}}+\Delta_{\{0,2\}}+\Delta_{\{0\}}+\cdots+\Delta_{\{0\}}$. The left-degree vector is given by $(1,1,0,0,0,0,0,0)$ and our bijection assigns the base point of this cell to the base $\{\overline{1}, \overline{2}\}$.

Consider the leftmost triangle. The corresponding summand is given by $\Delta_{\{1\}}+\Delta_{\{2\}}+$ $\Delta_{\{2\}}+\Delta_{\{1\}}+\Delta_{\{1\}}+\Delta_{\{0,1,2\}}+\Delta_{\{0\}}+\Delta_{\{0\}}$. This cell is not zonotopal, and there is no base assigned to the cell. If we consider the cell to the top of it, the summand is given by $\Delta_{\{1\}}+\Delta_{\{2\}}+\Delta_{\{2\}}+\Delta_{\{1\}}+\Delta_{\{1\}}+\Delta_{\{1,2\}}+\Delta_{\{0,2\}}+\Delta_{\{0\}}$. The left-degree vector is given by $(0,0,0,0,0,1,1,0)$, and the base point of the cell is assigned to the base $\{\overline{6}, \overline{7}\}$.

\section{Acknowledgements}

The author would like to thank Alexander Postnikov, Richard Stanley, Criel Merino, Fabrizio Zanello and Hwanchul Yoo for useful discussions. The author would also like to 


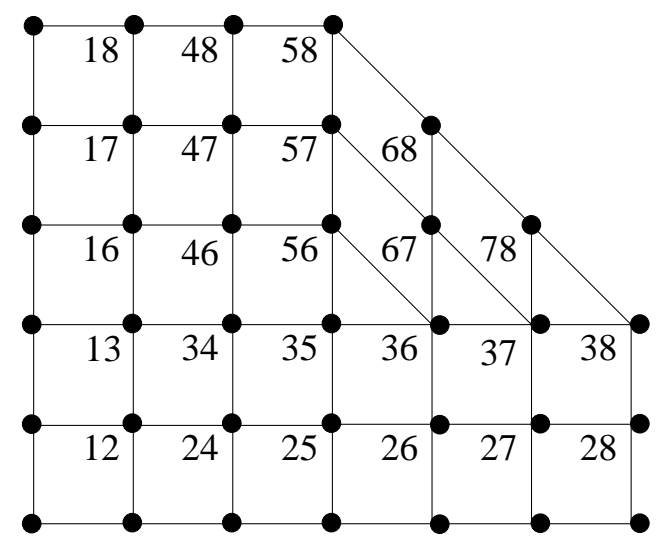

\begin{tabular}{|c|c|}
\hline$B$ & Externally passive elements \\
\hline 12 & $\emptyset$ \\
13 & 2 \\
16 & 2,3 \\
17 & $2,3,6$ \\
18 & $2,3,6,7$ \\
24 & 1 \\
25 & 1,4 \\
26 & $1,4,5$ \\
27 & $1,4,5,6$ \\
28 & $1,4,5,6,7$ \\
34 & 1,2 \\
35 & $1,2,4$ \\
36 & $1,2,4,5$ \\
37 & $1,2,4,5,6$ \\
38 & $1,2,4,5,6,7$ \\
46 & $1,2,3$ \\
47 & $1,2,3,6$ \\
48 & $1,2,3,6,7$ \\
56 & $1,2,3,4$ \\
57 & $1,2,3,4,6$ \\
58 & $1,2,3,4,6,7$ \\
67 & $1,2,3,4,5$ \\
68 & $1,2,3,4,5,7$ \\
78 & $1,2,3,4,5,6$ \\
\hline
\end{tabular}

Figure.3 \& Table.1 - A lexicographical subdivision of $P_{\mathcal{M}}$ and a table of bases in $\mathcal{M}$, where the bars of the ground set $\{\overline{1}, \ldots, \bar{n}\}$ is omitted for convenience.

thank Jose Soto for his advice on transversal matroids, and David Speyer for his advice on fine mixed subdivisions.

\section{References}

[1] M. Boij, J. Migliore, R. Mirò-Roig, U. Nagel, and F. Zanello: "On the shape of a pure $O$-sequence", Memoirs of the American Mathematical Society Series 218, American Mathematical Society (2012).

[2] M.K. Chari: Matroid inequalities, Discrete Math. 147 (1995), 283-286.

[3] M.K. Chari: Two decompositions in topological combinatorics with applications to matroid complexes, Transactions of the American Mathematical Society. 349 (1997), no. 10, 3925-3943. 
[4] P. Hall: On Representatives of Subsets, Journal of the London Mathematical Society. 10 (1935), 26-30.

[5] T. Hausel and B. Sturmfels: Toric hyperKäler varieties, Doc. Math. 7 (2002), 495534 .

[6] T. Hibi: What can be said about pure O-sequences?, J. Combin. Theory Ser. A 50 (1989), no. 2, 319-322.

[7] C. Merino: The chip firing game and matroid complexes, Discrete models: combinatorics, computation, and geometry (2001), Discrete Math. Theor. Comput. Sci. Proc., AA, Maison Inform. Math. Discrete. (MIMD), Paris (2001), 245-255.

[8] J.G. Oxley: "Matroid theory", Oxford University Press (2006).

[9] N. Proudfoot: On the h-vector of a matroid complex, unpublished note (2002).

[10] A. Postnikov: Permutohedra, associahedra, and beyond, International Mathematics Research Notice. 6 (2009), 1026-1106.

[11] J. Schweig: On the h-Vector of a Lattice Path Matroid, Electronic Journal of Combinatroics. 17 (2010), no. 1, N3.

[12] R. Stanley: "Combinatorics and commutative algebra", Second Ed., Progress in Mathematics 41, Birkhäuser Boston, Inc., Boston, MA (1996).

[13] E. Stokes: "The $h$-vectors of matroids and the arithmetic degree of squarefree strongly stable ideals", Ph.D. Thesis, University of Kentucky (2008).

[14] H. Tái Hà, E. Stokes, F.Zanello: "Pure O-sequences and matroid h-vectors", Annals of Combinatorics, to appear.

[15] N. White, Ed.: "Theory of Matroids", Encyclopedia of Mathematics and Its Applications 26, Cambridge Univ. Press, Cambridge (1986).

[16] N. White, Ed.: "Matroids Applications", Encyclopedia of Mathematics and Its Applications 40, Cambridge Univ. Press, Cambridge (1992). 